\title{
Análisis de la resistencia mecánica del cuerno del toro. Influencia del enfundado
}

\section{Analysis of the Mechanical Resistance of the Lidia Bull Horns. Influence of the Sheathing}

\section{Análise da resistência mecânica do corno do touro. Influência do revestimento}

\author{
Juan Manuel Lomillos ${ }^{1 \otimes}$, José Ramiro González-Montaña ${ }^{2}$, Marta Elena Alonso ${ }^{3}$
}

1 Departamento de Producción y Sanidad Animal, Salud Pública Veterinaria y Ciencia y Tecnología de los Alimentos. Facultad de Veterinaria. Universidad Cardenal Herrera-CEU. C/ Tirant lo Blanc, 7. 46115 Alfara del Patriarca - Valencia (Spain).

2 Departamento de Medicina, Cirugía y Anatomía Veterinaria. Facultad de Veterinaria de León. Universidad de León. Campus de Vegazana s/n. 24071 León (Spain).

3 Departamento de Producción Animal. Facultad de Veterinaria de León. Universidad de León. Campus de Vegazana s/n. 24071 León (Spain).

Recibido: 17 de febrero de 2021 Corregido: 30 de noviembre de 2021 Aceptado: 1 de diciembre de 2021

\begin{abstract}
Resumen
La cornamenta del toro de lidia es la parte anatómica más delicada de este animal, dado que su carácter ofensivo y estresable hace que sufra lesiones traumáticas durante el año previo a la lidia. Actualmente, se protege el cuerno con una funda de fibra de vidrio, con el objetivo de proteger su integridad. Con este trabajo, se pretende estudiar la resistencia mecánica del cuerno del toro de lidia; se analiza la posible influencia del enfundado. Para ello, se ha recogido cuernos de 50 toros de lidia de 4 a 5 años; 25 de ellos habían sido enfundados 6 meses antes de su lidia. Se ha analizado la resistencia mecánica del cuerno: cepa, pala y pitón; se utiliza el equipamiento Instron Electropulse E10000, siguiendo una metodología similar a la empleada por Horcajada et al. (2009). Se realiza tres pruebas de resistencia mecánica: compresión directa, tracción indirecta y flexo-tracción. Todas reflejaron una alta resistencia del cuerno, sin diferencias entre sus partes. Se observa un efecto beneficioso del enfundado, favorece el espesor del estuche de queratina en la cepa del cuerno; el cual fue mayor en los cuernos de los animales enfundados. Además, se comprueba una mayor resistencia de los cuernos enfundados en la prueba de tracción indirecta. Por otra parte, los cuernos de mayor longitud y diámetro resistieron presiones más altas, absorbiendo el hueso cornal, mayoritariamente, la presión ejercida.
\end{abstract}

Palabras clave: raza de lidia, cuernos, resistencia mecánica.

\begin{abstract}
The bull horns are the most delicate anatomical part of this animal since its offensive and easily stressful character causes them to suffer traumatic injuries during the year prior to the show. Currently, the horn is protected with a fiberglass sleeve to protect its integrity. The present work studies the mechanical resistance of bull horns, analyzing the possible influence of the sheath on them. For this, horns have been collected from 50 fighting bulls, 4 to 5 years old, 25 of which had been sheathed 6 months before their show. The mechanical resistance of horns has been analyzed in the distal, middle, and proximal parts, using the Instron Electropulse E10000 equipment, following a
\end{abstract}

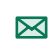

Autor de correspondencia: juan.lomillos@uchceu.es 
methodology like that used by Horcajada et al. (2009). Three mechanical resistance tests were performed: direct compression, indirect traction, and flexo-traction; they showed high resistance of the horn, without differences between its different parts. A beneficial effect of the sheath is observed, favoring a greater thickness of the keratin envelope at the proximal part of the horn; the thickness was greater in the sheathed horns. In addition, greater resistance of the horns sheathed is observed in the indirect traction test; the horns of greater longitudinal and diameter resisted the highest pressures, and the corneal bone mostly absorbed the pressure exerted.

Keywords: Lidia cattle, horns, mechanical resistance.

\begin{abstract}
Resumo
Os cornos do touro de lide são a parte anatômica mais delicada deste animal, pois sua natureza ofensiva e estressante faz com que sofra lesões traumáticas durante o ano anterior à luta. Atualmente, o corno é revestido com uma capa de fibra de vidro, a fim de proteger sua integridade. Com este trabalho, pretende-se estudar a resistência mecânica do corno do touro de lide; e analizar a possível influência do revestimento. Para isso, foram coletados cornos de 50 touros de lide entre 4 e 5 anos de idade; 25 deles foram embainhados 6 meses antes de seu evento. A resistência mecânica do corno foi analisada: base, zona intermedia e ponta do revestimento do corno, utilizando o equipamento Instron Electropulse E10000, seguindo metodologia semelhante à utilizada por Horcajada et al. (2009). Os três testes de resistência mecânica realizados: compressão direta, tração indireta e flexo-tração refletiram uma alta resistência do corno, sem diferenças entre suas partes. Observa-se um efeito benéfico do revestimento, que favorece a espessura do envoltório de queratina na tensão do corno; que era maior nos cornos dos animais com revestimento. Além disso, verifica-se uma maior resistência dos cornos revestidos no teste de tração indireta. Por outro lado, os cornos de maior comprimento e diâmetro resistiram as pressões mais elevadas, absorvendo o osso cornual, principalmente, a pressão exercida.
\end{abstract}

Palavras-chave: raça de lide, cornos, resistência mecânica.

\title{
Introducción
}

La cornamenta de los bóvidos es una producción epidérmica, situada a ambos lados del testuz y sustentada en el saliente óseo del hueso frontal (Calvo, 2005; Fernández, 2009; Martín, 1984). Es una estructura anatómica susceptible de sufrir diversas patologías (Sotillo et al., 1996) y, sobre todo, lesiones traumáticas en el caso de animales con un temperamento fácilmente estresable, como es el caso de los toros de lidia (Alonso et al., 2016).

La encornadura del toro sufre un riesgo de deterioro, fundamentalmente en el último año de vida, a consecuencia de potenciales peleas, roces, contactos o golpes con el suelo, con árboles, el vallado, comederos o las paredes de las mangas o corrales de manejo (Aparicio et al., 2000). Por ello, en la década de los '60, se popularizó, entre las ganaderías del sur de España, el uso de planchas finas de plomo para el recubrimiento o protección de los cuernos. Estas fundas consistían en láminas muy finas, las cuales se moldeaban alrededor del pitón. El método ofrecía gran protección frente al rascado contra el suelo o las encinas, pero el metal lesionaba las puntas de los pitones cuando los toros peleaban.

Posteriormente, en la década de los '80, se cambió el plomo por aluminio y las láminas por un recubrimiento que se adhería al cuerno con pegamento. Estas variaciones no producían lesiones, pero el artilugio se desprendía fácilmente (Lomillos et al., 2013).

En los '90, se opta por sustituir el aluminio por PVC (policloruro de vinilo), mucho más ligero y manejable, pero este material causaba maceraciones y, en algunos casos, putrefacciones en el tejido córneo (Gómez, 2011). 


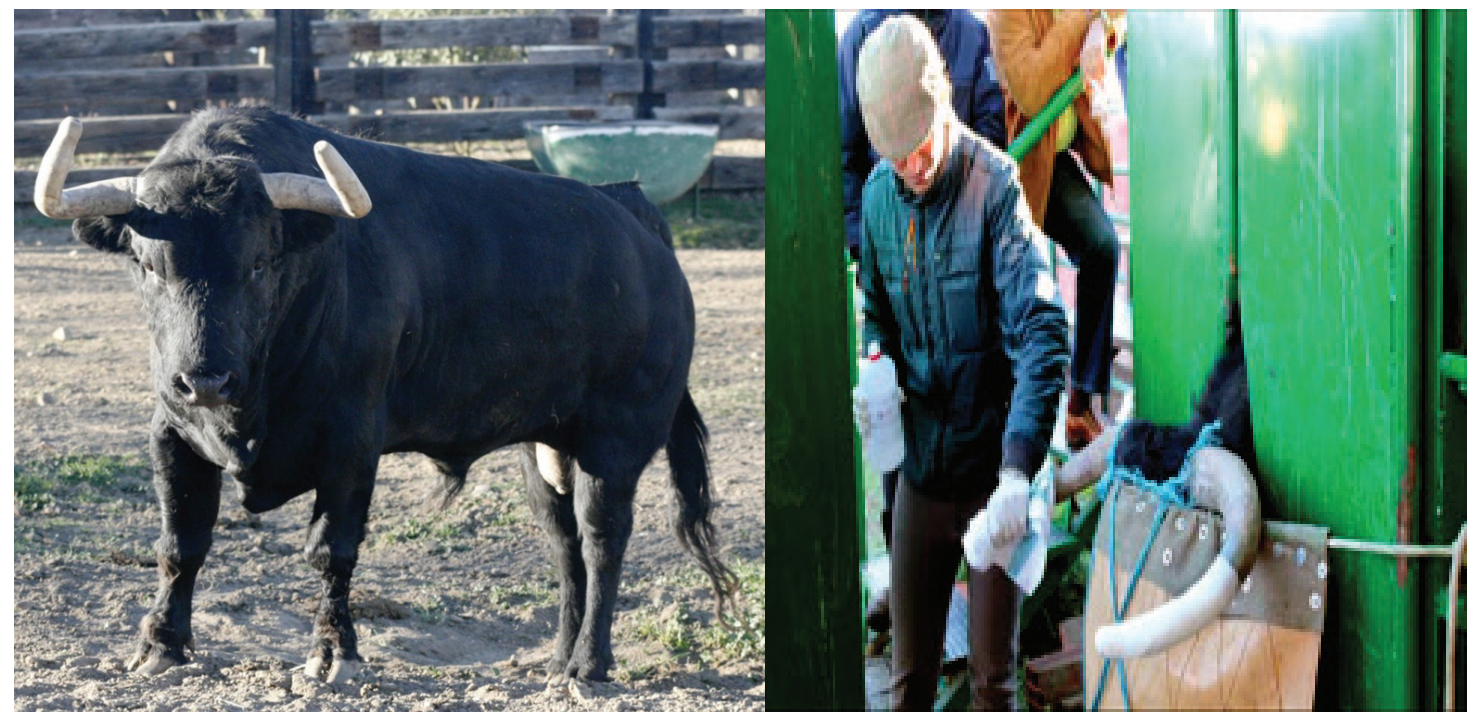

Figura 1. Macho de Lidia enfundado (Fotografías: Julio Cesar Sánchez, 2018).

Actualmente, se usa un vendaje de fibra de vidrio, fácil de manejar, poroso y que se endurece rápidamente por polimerización con el agua, proporcionando buena consistencia (Figura 1). La técnica consiste en inmovilizar al animal en el cajón de contención y envolver el cuerno con este vendaje para protegerlo de cualquier agresión o roce. La parte distal del cuerno, es decir el pitón, se refuerza, en muchos casos, con un material más duro, tubos metálicos o similares, con el fin de disminuir el desgaste de la zona apical (Pizarro et al., 2008a, Pizarro et al., 2008b).

La encornadura queda aumentada de grosor por la funda y el pitón romo, lo cual disminuye el efecto de las cornadas entre animales en un 90\% y, además, mejora su manejo para vacunaciones, desparasitaciones y otros tratamientos, puesto que se reduce los riesgos de deterioro de las defensas al pasar los animales por las mangas de manejo (Lira, 2008).

Existe un debate sobre el uso legítimo de las fundas ante la evidente manipulación de los cuernos en los momentos de la colocación y retirada del vendaje. (REAL DECRETO 176/1992). Igualmente, se desconoce cómo afecta este tipo de manipulación del cuerno a su estructura y, por ende, a su dureza. Algunos autores han observado una necrosis de la dermis cornal, con pérdida de estructura ósea y aparición de espacios huecos en el interior del cuerno, los cuales podrían reducir su resistencia (Gómez, 2009). Se ha realizado algunos estudios sobre el efecto de este enfundado sobre la dureza superficial del cuerno, que reflejan conclusiones dispares (Horcajada et al., 2009; Pizarro et al., 2008a; Pizarro et al., 2008b; Pizarro et al., 2009; Salamanca, 2009). Por ello, el objetivo de este trabajo consiste en arrojar luz sobre el efecto del enfundado sobre la resistencia mecánica del cuerno, con una amplia muestra de animales y una metodología de análisis adecuado para establecer conclusiones sólidas. 


\section{Material y métodos}

Se analizó el cuerno derecho de 50 toros de lidia de 3 a 5 años, 25 de ellos habían sido enfundados 6 meses antes de su sacrificio.

Se realizó pruebas mecánicas utilizando el equipamiento Instron Electropulse E10000, siguiendo una metodología similar a la empleada por Horcajada et al. (2009). Se trata de una máquina de ensayos hidráulica, homologada acorde con la norma UNE- EN 12390-4:2000, de $2000 \mathrm{kN}$ de capacidad máxima de carga (200 tn), fabricante IIC, SA, modelo H-2000-CO, utilizada, habitualmente, para el ensayo y control de calidad de hormigones.

La prensa aplica una fuerza de forma axial sobre el elemento por ensayar hasta su rotura; o bien, hasta el paro de la misma por parte del usuario. La medida de la fuerza total aplicada es recogida por un data-logger integrado en el dispositivo que se controla a través de una aplicación informática; el cual, además, gestiona de manera automática el grupo hidráulico. Se decidió realizar los siguientes ensayos:

Prueba 1- Ensayo de compresión directa (norma UNE EN-12390-3:2009): la prensa hidráulica aplica, a velocidad constante, una carga axial directa centrada sobre el cuerno (Figura 2).

Prueba 2 - Ensayo de tracción indirecta o ensayo brasileño (norma UNE-EN 12390-6:2010): el cuerno se dispone sobre el dispositivo adaptador de tracción indirecta (Figura 3). La prensa aplica la carga de manera constante por la parte superior del adaptador que la transmite al cuerno en toda su longitud (a diferencia del ensayo siguiente, en el cual la carga se aplica únicamente en dos puntos). La rotura, en este caso, se produce a lo largo de la directriz de la pieza sometida al ensayo.

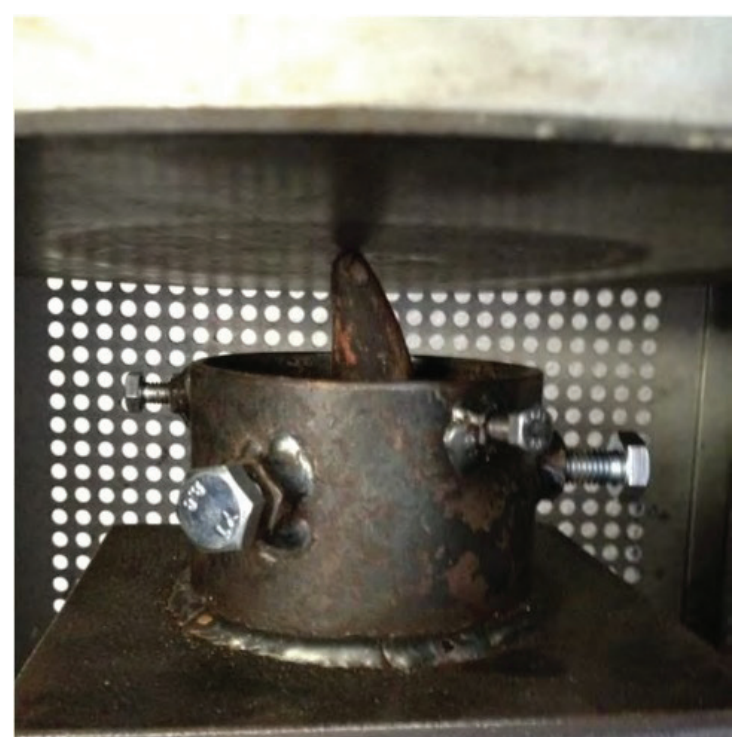

Figura 2. Ensayo de compresión directa.

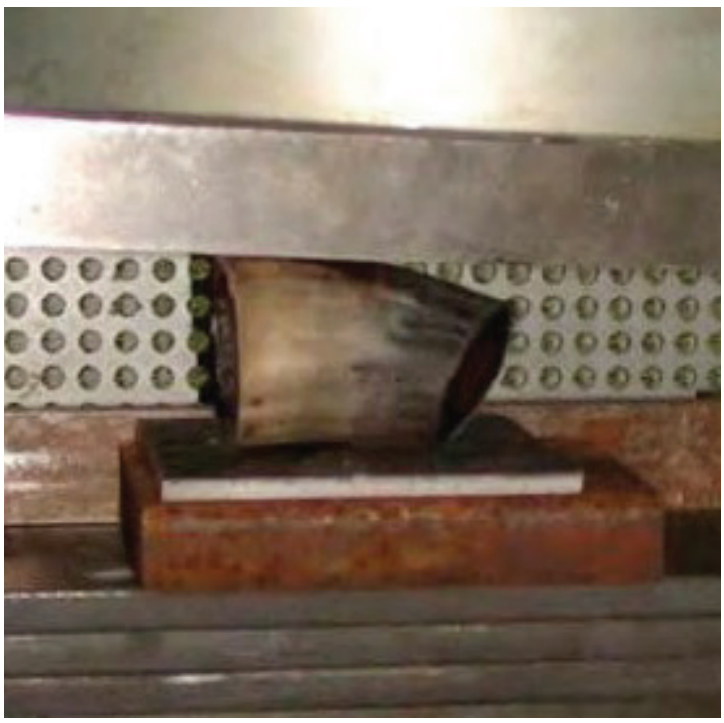

Figura 3. Adaptador para ensayo de flexo-tracción modificado con cuerno en decúbito lateral. 
Prueba 3 - Ensayo de flexo-tracción (norma UNE-EN 12390-5:2009): el cuerno se dispone sobre el dispositivo adaptador de flexo-tracción. La prensa aplica la carga, de manera constante, por la parte superior del adaptador que la transmite en dos puntos situados en el tercio central del cuerno. Entre dichos puntos, se produce un estado de cargas o solicitaciones denominado flexión pura, donde el cuerno se agota y rompe únicamente debido a la flexión a la que es sometido. Habitualmente, la zona donde se produce la rotura es la traccionada, situada, en este caso, en la parte media inferior del cuerno (Figura 4).

Se tomaron medidas exactas de la dimensión de los cuernos: longitud (L) del macizo córneo, L del cuerno, diámetro $(\varnothing)$ del cuerno en la cepa, $\varnothing$ del hueso en la cepa y espesor (E) del estuche de queratina en la cepa y su peso, con el objetivo de estudiar su posible efecto en las pruebas.

Tras diferentes pruebas, para definir las dimensiones adecuadas, para realizar los cortes y secciones, los cuernos fueron cortados en 3 partes, la primera a una longitud de $9,5 \mathrm{~cm}$ del pitón, la segunda de $5 \mathrm{~cm}$ para la prueba 2 y la tercera de $25 \mathrm{~cm}$ para la prueba 3.

Todos los datos fueron procesados mediante el programa SPSS. Se realizó un análisis de varianza (ANOVA) de una vía, considerando grupo 0 los animales no enfundados y grupo 1 los enfundados.

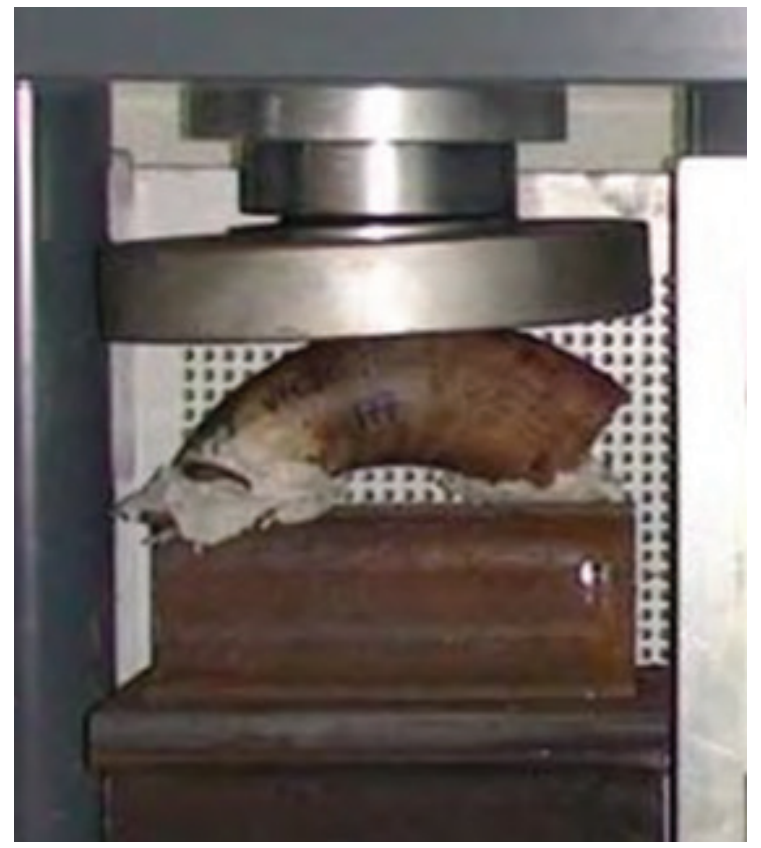

Figura 4. Adaptador para ensayo de flexo-tracción modificado con cuerno en apoyo sobre la curvatura mayor.

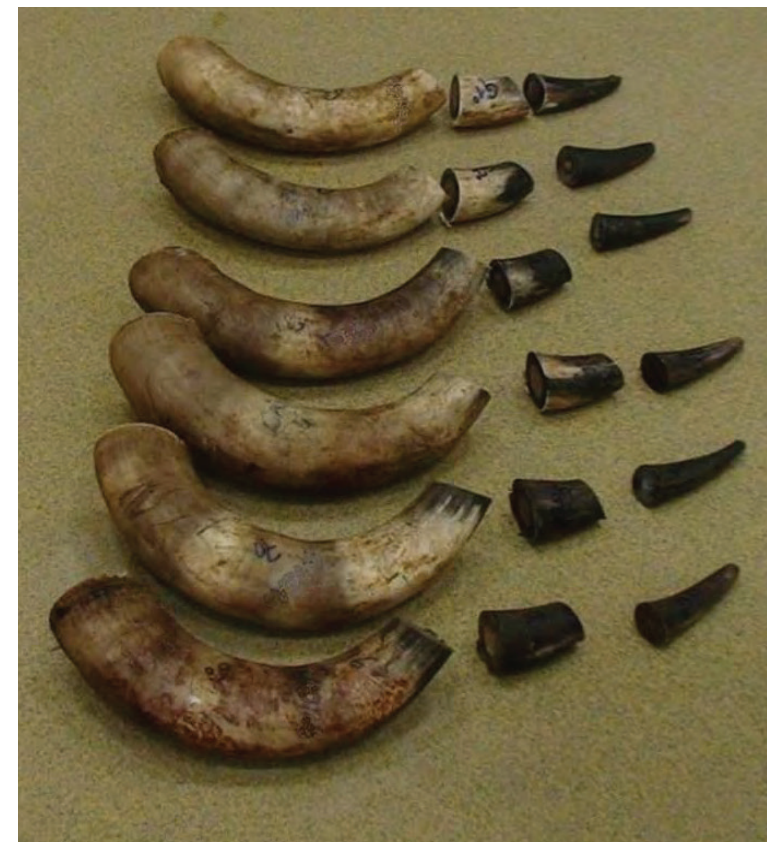

Figura 5. Secciones preparadas para las tres pruebas. 


\section{Resultados y discusión}

Tras la realización del ANOVA, de una vía, entre los distintos parámetros biométricos recogidos en los diferentes cuernos empleados en las pruebas, se comprobó que no existían diferencias significativas entre los dos grupos en estudio, salvo el espesor del estuche de queratina en la cepa, que fue mayor en los cuernos de los animales enfundados.

Tabla 1. ANOVA de los datos biométricos medidos, expresados en mm.

\begin{tabular}{lllllllll}
\hline & Media 0 & Media 1 & Des. Est. 0 & Des. Est. 1 & df df & F & P \\
\hline L macizo córneo & 96,308 & 93,286 & 5,544 & 6,566 & 1 & 32 & 1,906 & 0,177 \\
L cuerno & 778,538 & 769,952 & 74,043 & 102,395 & 1 & 32 & 0,543 & 0,466 \\
Ø cuerno cepa & 49,462 & 51,214 & 4,390 & 3,273 & 1 & 32 & 0,897 & 0,351 \\
Ø hueso cepa & 39,154 & 38,252 & 7,537 & 7,092 & 1 & 32 & 1,111 & 0,300 \\
E queratina cepa & $10,231^{*}$ & $14,167^{*}$ & 8,892 & 6,545 & 1 & 32 & 0,001 & 0,981 \\
Peso & 1790,231 & 1711,570 & 356,778 & 269,071 & 1 & 32 & 0,002 & 0,963 \\
\hline
\end{tabular}

* Variable marcada es significativa a $\mathrm{p}<0.05$.

Los resultados de la resistencia mecánica, obtenida en las tres pruebas realizadas, se recogen en la Tabla 2. Se comprueba que tan solo hay diferencias significativas en el valor de la resistencia del cuerno en la prueba 2, siendo mayor en el grupo de animales enfundado a la fuerza de tracción indirecta (Grupo 1).

Tabla 2. ANOVA de los datos de las distintas Pruebas de resistencia realizados. Medias en kNewtons.

\begin{tabular}{cccccccccc}
\hline & \multicolumn{2}{c}{ Des. } & & Des. & df & df & F & p \\
\multicolumn{2}{c}{ Media 0 } & Media 1 & Est. 0 & & \multicolumn{2}{c}{ Est. 1 } \\
\hline Prueba 1 & 18,138 & 22,033 & 7,428 & 6,330 & 1 & 32 & 2,663 & 0,112 \\
Prueba 2 & $11,000^{*}$ & $14,475^{*}$ & 3,419 & 4,943 & 1 & 31 & 4,879 & 0,035 \\
Prueba 3 & 88,500 & 90,124 & 12,409 & 19,730 & 1 & 32 & 0,070 & 0,793 \\
\hline
\end{tabular}

* Variable marcada es significativa a $\mathrm{p}<0.05$.

Con los datos totales, obtenidos en las pruebas, se realizó una matriz de correlación lineal para comprobar si las medidas biométricas y los valores obtenidos, tras la prueba, presentaban alguna relación. 
Tabla 3. Matriz de correlación lineal entre parámetros obtenidos en la Prueba 1.

\begin{tabular}{lccc}
\hline & Prueba 1 & Prueba 2 & Prueba 3 \\
\hline L cuerno & $-0,01$ & $0,61^{*}$ & $-0,18$ \\
ó cuerno & 0,11 & $0,49^{*}$ & 0,22 \\
$\varnothing$ hueso & 0,05 & $0,59^{*}$ & $0,37^{*}$ \\
E queratina & $-0,06$ & $-0,41^{*}$ & 0,09 \\
peso & 0,31 & $0,71^{*}$ & 0,13 \\
\hline
\end{tabular}

Correlación marcada es significativa a $\mathrm{p}<0.05$.

En la prueba 2, se observa cómo los cuernos de mayor longitud, con una sección o diámetro mayor, resistieron presiones más altas antes de separarse la dermis que une el hueso y la capa de queratina. También, las secciones del cuerno de mayor diámetro de hueso registraron valores superiores, mientras los espesores menores de queratina se comportaron de modo inverso. Con base en los datos, se puede pensar que la capacidad de absorber la presión ejercida parece estar más en el hueso que en la capa de queratina del estuche córneo que lo envuelve; quizá porque esta parte es la que contiene más cantidad de minerales en su composición química (Cabanas et al., 1994). En la prueba 3, la única variable que resultó correlacionar con la resistencia a la flexotracción, resultó ser el diámetro del hueso, siendo más resistentes aquellos cuernos de hueso de mayor sección, pero sin diferencias entre los grupos estudiados.

Finalmente, se realizó una nueva Matriz de correlación entre los parámetros estudiados y la edad, el cual no arrojó diferencias significativas (Tabla 4).

Tabla 4. Matriz de correlación lineal entre la edad y las diferentes pruebas realizadas.

\begin{tabular}{ll}
\hline & Edad meses \\
\hline Prueba 1 & 0,04 \\
Prueba 2a & $-0,26$ \\
Prueba 3 & 0,16 \\
\hline
\end{tabular}

Nuestros resultados no se pueden contrastar con algún otro trabajo publicado, aunque, completan los datos aportados por Horcajada et al. (2009), los cuales miden el grado de deformación del cuerno tras la prueba de presión.

En nuestro caso, se observa una mayor resistencia a la prueba de tracción de los cuernos enfundados, lo cual conectaría con el trabajo de Horcajada et al. (2009) en el que registran una mayor deformación de los cuernos enfundados tras la prueba de presión; además, podría suponer una mayor flexibilidad de estos, como efecto de un enfundado que podría mantener las fibras de queratina más hidratadas, por ello, más flexibles. 
Con base en la información recogida, el enfundado parece ser un buen método de protección hacia el cuerno del toro de lidia, a pesar de que existen ciertos casos de posibles lesiones de cuernos, las cuales se dan con la funda instalada y podrían pasar desapercibidas y, finalmente, dar lugar a fracturas durante la lidia en la plaza de toros (Gómez et al., 2009).

\section{Conclusiones}

El espesor del estuche de queratina, en la cepa del cuerno, fue mayor en los cuernos de los animales enfundados. Se comprueba una mayor resistencia de los cuernos enfundados en la prueba de tracción indirecta, siendo los cuernos de mayor longitud y diámetro los que resistieron presiones más altas. Todo ello apunta un efecto beneficioso del enfundado, que aumentaría su resistencia.

\section{Agradecimientos}

Gracias al Dr. Hernán Gonzalo del Departamento de Ingeniería Civil, Área de Ingeniería e Infraestructura de los Transportes de la Universidad de Burgos; al Dr. Andrés Valdés del Departamento de Ingeniería y Ciencias Agrarias de la Universidad de León y a la empresa ITMA. Este trabajo ha sido parcialmente financiado por la Asociación de Veterinarios Taurinos de España (AVET).

\section{Referencias}

Alonso, M.E., Lomillos, J.M., González, J.R. (2016). La cornamenta del toro de lidia análisis de su integridad $y$ efecto del enfundado. León: EOLAS Ediciones.

Aparicio-Sánchez, G. (1960). Exterior de los grandes animales domésticos. Córdoba: Imprenta Moderna.

Aparicio, J.B., Peña, F., Barona, L.F. (2000). Estudio de las encornaduras del Toro de Lidia. Ed. Junta de Andalucía. Córdoba.

Cabanas, J.M., Gualda, M.J., Rosa, M. y del Pino, J. (1994). Estudio de la composición mineral de las astas del Toro de lidia. I Congreso Mundial de Veterinaria Taurina. Zaragoza.

Calvo, L.A. (2005). Escuela gráfica de toros. Valladolid: Colegio Oficial de Veterinarios de Valladolid.

Fernández, J. (2009). Atlas gráfico de pelajes y encornaduras. Curso básico de Espectáculos Taurinos. Madrid: Ed. Colegio de Veterinarios de Madrid.

Gómez, F.J., Fernández, F.J., Pizarro, M., Carpintero, C.M., y Durán, J.M. (2009). Fractura de pitón durante la lidia, bipotéticamente asociado al enfundado de los cuernos. IX Simposio Nacional del Toro de Lidia. Zafra. Pp. 285-288.

Gómez, A. (2011). El enfundado del Toro de Lidia. VII Congreso Mundial Taurino de Veterinaria. Cáceres. Consejo General de Colegios Veterinarios de España. Pp 23-34.

Horcajada, F.J., Fernández, C., Ortuño, S., Pizarro, M. (2009). Diferencias físicas de comportamiento entre pitones enfundados y sin enfundar con vendas de resina de fibra de vidrio: prueba de compresión simple. IX Simposio Nacional del Toro de Lidia. Zafra. Pp. 237-242. 
Lira, F. (2008). Avances en el cuidado y protección de las defensas del Toro de lidia. Manual de manejo y nutrición del Toro de lidia. Tomo II. ITACYL Valladolid. Pp. 28-43.

Lomillos, J.M., Alonso, M.E. y Gaudioso, V. (2013). Análisis de la evolución del manejo en las explotaciones de toro de lidia. Desafíos del sector. Revista ITEA 109 (1), 49-68.

Martín, R. (1984). Estudio anatómico y biométrico de la cornamenta del toro de lidia. Medicina Veterinaria. Madrid. 1-11. Pp. 545-553.

Sañudo, C. (2009). Valoración morfológica de los animales domésticos. Ministerio de Medio Ambiente y Medio Rural y Marino. Madrid.

Pizarro, M., Carceller, H., Alonso, R., Horcajada, J., Hebrero, C. (2008a). Utilización de fundas en cuernos I: Colocación e incidencia en el reconocimiento y comportamiento. VI Congreso Mundial Taurino de Veterinaria. Murcia. Pp. 175- 178.

Pizarro, M., Alonso, R., Ortuño, S. y Fernández, C. (2008b). Utilización de fundas en cuernos II: Posible modificación de la estructura y consistencia. VI Congreso Mundial Taurino de Veterinaria. Murcia. Pp. 179-182.

Pizarro, M., Horcajada, F.J., Fernández, C. y Ortuño, S. (2009). Diferencias estructurales entre pitones enfundados y sin enfundar con vendas de resina de fibra de vidrio. IX Simposio Nacional del Toro de Lidia. Zafra. Pp. 285-288.

Real Decreto 176/1992, de 28 de febrero, por el que se aprueba el Reglamento de espectáculos taurinos. BOE núm. 56, de 5 de marzo de 1992. Pp. 7570 - 7583.

Salamanca, P. (2009). Análisis de la dureza en cuernos de machos de Lidia de tres y cuatro años de edad comparando el factor encaste. IX Simposio Nacional del Toro de Lidia. Zafra. Pp. 263-274.

Sotillo, F., Ramírez, A.R., Sotillo, J.L. (1996). Biotipología del Toro de Lidia. En: Producciones equinas y de ganado de Lidia, Cap. XV.Zootecnia, bases de producción animal, tomo XI. Ed. Mundiprensa. Madrid. 